\title{
LAS NUEVAS TECNOLOGIAS DE LA INFORMACION Y LAS COMUNICACIONES Y EL DESARROLLO HUMANO SUSTENTABLE
}

\author{
Aquiles Bedriñana Ascarza* \\ E-mail: aquiles@telefonica.net.pe
}

\begin{abstract}
RESUMEN
Vivimos la revolución tecnológica más importante experimentada por la humanidad. Las Nuevas Tecnologías de Información y Comunicación (NTIC) son hoy producidas ya mayoritariamente por las grandes transnacionales que siguen a la demanda con mayor poder adquisitivo. Las prioridades y necesidades de los países pobres no merecen atención por limitaciones en el financiamiento de la investigación, desarrollo e innovación $(\mathrm{I}+\mathrm{D}+\mathrm{I})$. El hecho de que dos terceras partes de la humanidad no hayan obtenido de las nuevas tecnologías mejoras sustanciales en sus condiciones de vida y algunos las hayan empeorado, tampoco alienta.
\end{abstract}

Cuando el desarrollo ha de basarse principalmente en la capacidad para procesar y aplicar conocimiento en redes internacionales de producción e intercambio, la amenaza de un mundo todavía más desigual $\mathrm{y}$ fraccionado no es ilusoria.

El presente trabajo trata de explicar que la llamada brecha digital no es, sin embargo, el problema, sino el resultado de otras brechas más importantes en capital humano, capital social, capacidades institucionales, gobernabilidad democrática, etc. Ellas son las que impiden que los países pobres y el mundo aprovechen las inmensas oportunidades que representan las NTIC.

En el presente trabajo, se trata de dar respuesta, en alguna medida a las siguientes interrogantes:

1. ¿Pueden las NTIC tener impactos eficaces en la reducción de la pobreza?

2. ¿Pueden las NTIC mejorar la calidad y equidad de la educación?

3. ¿Estos impactos pueden notarse en el corto y mediano plazo?

Palabras Clave: NTIC, Índice de Desarrollo Humano Sustentable (IDHS), brecha digital, capital humano, calidad y pertinencia, desarrollo de las capacidades humanas.

\begin{abstract}
Nowadays we are living in the most important technological revolution experimented by the humanity. The New Information and Communication Technologies (NICT) are already produced by the biggest multinational companies that follow the demand which has more spending capacity. The priorities and necessities of poorest countries do not deserve attention because of limitations in the financing of research, development and innovation $(\mathrm{R}+\mathrm{D}+\mathrm{I})$. The fact that two third - parts of humankind have not obtained from the new technologies substantial improvements in their lives and some of them go worse
\end{abstract}

* Doctor en Educación, Magíster en Ingeniería de Sistemas y MBA por la Universidad de Cali, Colombia; Profesor Principal e Investigador permanente de la Unidad de Investigación y Decano de la Facultad de Ciencias Administrativas de la UNMSM. Profesor de Post Grado de la UNMSM y de otras universidades. 
does not encourage. When development has to be based mainly on the capacity to process and to apply the knowledge in international networks of production and interchange, the threat of an even more unequal and divided world is not false.

This work tries to explain that the so called digital gap is not problem, but the result of other more important gaps in human capital, social capital, institutional capacities, democratic governability, etc. They are make difficult the poorest countries of the world to take advantage of the immense opportunities that represent the NICT.

In this work, we try to answer in some way to the following questions:

1. Can the NICT have effective impacts in reduction of the poverty?

2. Can the NICT improve the quality and equitity of education?

3. Can these impacts be noticed in the short and medium term?

Key words: NICT, Human Sustainable Development Index (HSDI), digital gap, human capital, quality and pertinence, human capacities development.

\section{INTRODUCCIÓN}

Desde varias décadas se viene planteando una correlación positiva o negativa entre la integración de las NTIC al sistema educativo y la reducción de la pobreza. Diversos autores plantean que las NTIC contribuyen al desarrollo económico de las naciones, fundamentalmente en el caso de la economía de los países desarrollados, pero también coinciden en que el desarrollo de las NTIC tiende a aumentar la desigualdad respecto a los países subdesarrollados.

Hacia fines de 1980, Amartya Sen ${ }^{1}$ desarrolla para las Naciones Unidas el concepto de Desarrollo Humano y su Índice de Desarrollo Humano Sustentable (IDH). A partir de 1990 las Naciones Unidas publican anualmente el Informe de Desarrollo Humano a nivel mundial.

El Índice de Desarrollo Humano (IDH), calculado por el PNUD, se conforma a partir de indicadores demográficos, educacionales y económicos dirigidos a medir no solamente la riqueza acumulada por la sociedad, sino la calidad de vida de los seres humanos y refleja enormes y crecientes desigualdades entre desarrollo y subdesarrollo. El desarrollo humano progresivamente ha ido incorporando muchas variables como crecimiento económico, educación, salud, medio ambiente, participación política, equidad de género, acceso a las NTIC, derechos humanos, democracia, etc.

Por otra parte, desarrollo sustentable es aquel que atiende a las necesidades de las generaciones presentes sin comprometer las necesidades de las futuras generaciones ${ }^{2}$. La definición anotada da a entender que el desarrollo sustentable comprende, al menos, dos perspectivas que se pueden sintetizar en dos objetivos: el objeti- vo actual, que es el mejoramiento de la calidad de vida de todos los habitantes; y el objetivo futuro, no menos importante, que consiste en no comprometer el futuro de las próximas generaciones.

En los países en vías de desarrollo como el nuestro, es importante estudiar si los beneficios generados por las NTIC llegarán realmente a cubrir las necesidades de los pobres y si podrán ayudar a reducir sus privaciones. Un mejor acceso a la educación, la salud e información sobre mercados agrícolas o a servicios del gobierno vía Internet, tienen un mayor valor para la gente de escasos recursos, debido a que no tienen acceso a los medios de comunicación tradicionales por sus altos costos.

Ricardo Monge ${ }^{3}$, economista que redactó la estrategia digital de Costa Rica, recuerda que el papel de las NTIC en el desarrollo sólo puede entenderse si se revisa el rol que la información ha cumplido en la humanidad. Sobre este tema, él menciona a Richard Heeks ${ }^{4}$, quien hizo notar que este papel puede dividirse en dos partes: el de la información en los procesos y el de la información en los resultados. En el primer caso, el impacto en el desarrollo tiene que ver con el hecho de convertir datos en información valiosa para el usuario y con la comunicación como mecanismo para transferir los datos desde una fuente hasta un receptor. En el segundo caso, el impacto de la información en el desarrollo está asociado con el aprendizaje entendido como la metamorfosis de la información dentro del conocimiento y con la toma de decisiones y acciones. En síntesis, es el apoyo de las NTIC en el aprendizaje y en la toma de decisiones y acciones lo que se constituye en la verdadera contribución al desarrollo. 
La economía basada en el conocimiento es totalmente diferente a la economía del siglo XX. En el mundo moderno, las economías más prósperas se basan en las habilidades de los ciudadanos, empresas, organizaciones, gobierno y sector privado para generar, almacenar, recuperar, procesar y transmitir informaciones; funciones que son aplicables a todas las actividades del ser humano. Por ello, Robert Atkinson ${ }^{5}$ indica que la premisa de una buena economía del conocimiento se basa en los seres humanos que utilizan datos, es decir, información útil.

Diversos estudios han documentado los beneficios sociales y ganancias económicas derivados del acceso y uso de las NTIC. Entre los beneficios se encuentran, por ejemplo, una mejor calidad de vida de las personas gracias a la optimización en la educación, la salud y la participación en las decisiones políticas, a pesar de que son más difíciles de cuantificar.

Un ejemplo vinculado con sectores deprimidos de la sociedad podemos encontrarlo en el caso de algunos telecentros comunitarios, que son lugares de acceso a las NTIC para los individuos y la comunidad reduciendo la brecha digital.
Las NTIC pueden potenciar a nuestros pueblos para ser más competitivos en la economía global, y garantizan la entrada de los individuos como entes productivos y activos en la nueva sociedad de la información en la que vivimos y viviremos durante las próximas décadas.

\section{NTIC Y DESARROLLO HUMANO}

El PNUD dedicó su informe anual correspondiente al año 2001 al análisis de las relaciones entre tecnología y desarrollo humano. El informe hace una relación de las vinculaciones que se dan entre las innovaciones tecnológicas y el desarrollo humano, que se plasma en el gráfico siguiente (Ver Gráfico 1). De acuerdo con su planteamiento, no hay duda que las tecnologías inciden positivamente en el desarrollo humano. En primer lugar, porque las innovaciones elevan de manera directa la capacidad humana. En segundo lugar, porque constituyen un medio para lograr el desarrollo humano debido a sus repercusiones en el crecimiento económico, gracias al aumento de productividad que generan. Las innovaciones tecnológicas son una expresión de la potencialidad humana; por consiguiente, el desarrollo humano y los avances tecnológicos se refuerzan mutuamente.

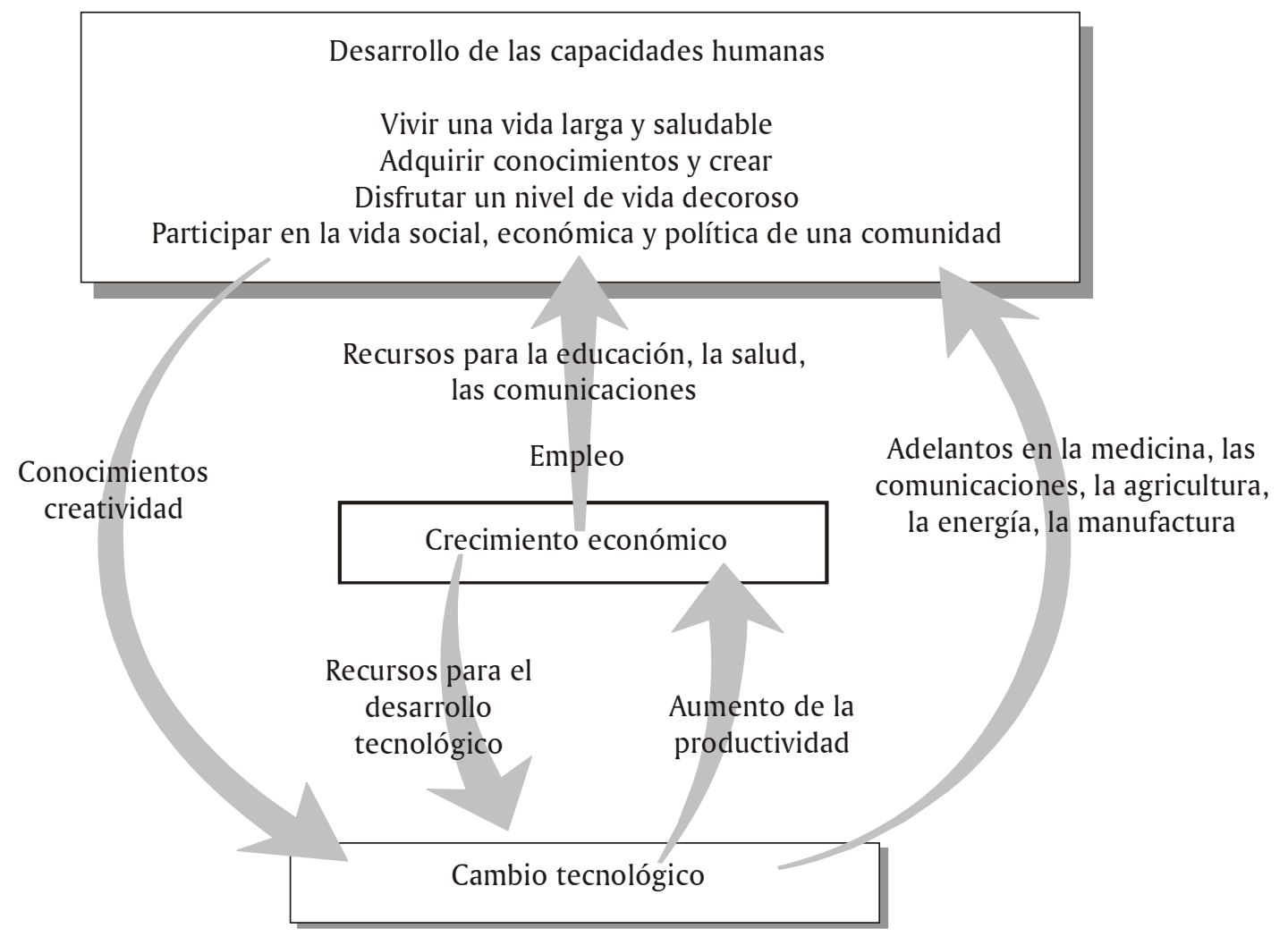

Gráfico 1. Vínculos entre la tecnología y el desarrollo humano. 
Aunque luego se hacen consideraciones complementarias, conviene destacar que este punto de partida resulta confuso. Lo decisivo no es afirmar la potencialidad teórica de las nuevas tecnologías, sino analizar cuándo y en qué condiciones su aplicación se plasma realmente en procesos de mejora del desarrollo humano. La potencialidad de transformar positivamente la realidad se encuentra en toda tecnología, pero preguntarnos por su incidencia en el desarrollo humano supone descifrar la compleja red de relaciones que hacen que se produzca o no. Los vínculos entre tecnología y desarrollo humano deben establecerse no de manera lógica, sino asegurando que realmente esas potencialidades se traducen en resultados de aumento de las capacidades de las personas. La tecnología no es relevante para el desarrollo humano si no se convierte en aumento de las capacidades de las personas. La pregunta es averiguar bajo qué contexto concreto -reglas de juego, instituciones, correlación de fuerzas, etc.- se produce esa relación benéfica. Si no se hace así, de alguna manera se está cayendo en el mismo error de la economía convencional que partía de la creencia de que el crecimiento económico era suficiente para erradicar la pobreza o conseguir el desarrollo. El enfoque del desarrollo humano ha criticado fuertemente este presupuesto por desconocer que crecimiento no suponía por sí mismo mejoras para el desarrollo de las personas. Ahora hay que evitar caer en la complacencia de una vinculación positiva per se entre tecnología y desarrollo humano.

Por eso en el Gráfico 1, que pretende mostrar las relaciones entre el desarrollo humano y la tecnología, falta introducir los mecanismos o procesos que aseguran que las personas y las sociedades acceden a su uso y deciden cómo emplearlas según sus preferencias; o, por el contrario, los obstáculos existentes que impiden su traducción en resultados. Si no se hace así, se elude un aspecto esencial de los vínculos reales: los problemas que plantea la difusión del conocimiento y el uso de las tecnologías en objetivos básicos de desarrollo humano.

\section{LAS NTIC EN LA EDUCACIÓN}

El dominio del conocimiento y en especial de las Nuevas Tecnologías de la Información y la Comunicación (NTIC), en el momento que vive hoy la humanidad, se ha convertido en instru- mento que contribuye a que la distancia que separa a los países desarrollados de los países subdesarrollados sea mayor, como resultado de las políticas capitalistas neoliberales globalizadas. A ello deben añadirse las crecientes diferencias en la calidad de los servicios educacionales y en los recursos destinados a la educación al comparar a ambos grupos de países.

¿Qué nuevas exigencias plantean a la educación estas realidades? La respuesta, en primer término, exige establecer que para el sistema nacional de educación institucionalizado, ha constituido un permanente elemento de búsqueda el lograr una calidad y pertinencia que asegure las respuestas necesarias a las exigencias sociales; esto ha sido un permanente reto a lo largo de la historia educacional. A lo anterior habría que añadir que las realidades sociales y, en particular, las exigencias de la competitividad para insertarse en un mundo cada vez más interrelacionado e interdependiente (globalizado), aumentan la demanda social de respuestas por parte de la educación y de los educadores.

¿Es que la educación es muy lenta para adaptarse y cambiar? ¿O es que las necesidades sociales cambian muy rápidamente? Ambas preguntas a nuestro juicio tienen una respuesta positiva y en especial se destaca que, en las condiciones del mundo actual, los cambios impuestos por la globalización y favorecidos por las NTIC han hecho más dinámicas y variadas las respuestas exigidas a la educación, tanto en los niveles de formación básica como en los niveles educacionales encargados de la formación de la fuerza de trabajo calificada; de ahí que en los análisis de los expertos y dirigentes de políticas educativas el aspecto central del debate en los últimos años ha sido, es y será, la calidad de la educación ${ }^{6}$.

La educación es uno de los sectores en el que más se aplica las NTIC, pero se han identificado tres grandes problemas:

a) La gran mayoría de los planes y proyectos educativos se concentran en la educación básica y primaria y en pocos casos se pone énfasis en la educación superior. No se concentran suficientes esfuerzos en estimular estudios superiores sobre la Sociedad de la Información (SI), ni en crear nuevas carreras que formen profesionales adecuados para generar conocimientos adecuados. 
b) Las políticas y estrategias detectadas en los países de América Latina y el Caribe enfatizan el equipamiento informático y en menor grado la conectividad en las escuelas, y en pocos casos, la generación de contenidos. Equipar y conectar escuelas no significa per se generar educación para la SI: es necesario innovar los modelos pedagógicos para hacer frente a los retos que plantea el siglo XXI.

c) Las políticas nacionales detectadas como estímulos al desarrollo sustentable y al combate contra la pobreza, lejos de estar orientadas al empoderamiento de las comunidades, trabajan sobre problemas coyunturales.

\section{LECCIONES APRENDIDAS}

Las NTIC pueden y deben jugar un rol fundamental en el desarrollo social y económico de América Latina y el Caribe, pero éste es un rol reducido en las actuales circunstancias de globalización. La brecha digital, que usualmente se refiere a inequidades en el acceso a las NTIC, no es una causa sino un efecto de las brechas sociales, económicas y políticas existentes, en los niveles global, nacional y local.

Reducir la brecha digital no ayudará -por sí misma- a las comunidades a mejorar sus condiciones de vida, salir de la pobreza, o tener un acceso más equitativo a bienes y servicios; para que las NTIC constituyan un motor de desarrollo humano sustentable en el proceso de inserción al sistema educativo, será necesario diseñar una nueva pedagogía y capacitación de docentes en esta innovación.

\section{NOTAS}

1 Amartya Sen, Martha Nussbaum. (1993). Lives and Capabilities. Equality of What? - Social Science. 472 pág.

2 Gómez (1996). Pp. 15-16

3 Monge González, Ricardo y Chacón, Federico. La Brecha Digital. Digital Divide.

4 Entrevista a Robert C. Atkinson. Executive Director. Columbia Institute for Tele-information.

5 Op Cit.

6 González Corona, Jorge; Silesio, Santos y Martín Sabina, Elvira. (2001). Educación, Desarrollo y Crecimiento Económico. Congreso de Pedagogía 1999. Cuba, Palacio de Convenciones de la Habana. 